\title{
Streptococcus constellatus Causing Mycotic Aneurysm in A Patient with Diffuse Large B-Cell Lymphoma
}

\author{
Nadeem Sajjad Raja ${ }^{1}$, Albena Nikolova ${ }^{2}$, Theo Raudsepp ${ }^{3}$, Simon G Weston-Smith ${ }^{2}$, Annette Rumbold ${ }^{2}$, \\ Hizbullah Shaikh ${ }^{4}$, Sunil Gupta ${ }^{2}$ \\ ${ }^{1}$ Department of Microbiology, East Sussex Healthcare Trust, Hastings, United Kingdom \\ ${ }^{2}$ Department of Clinical Haematology, East Sussex Healthcare Trust, Hastings, United Kingdom \\ ${ }^{3}$ Department of Radiology, East Sussex Healthcare Trust, Hastings, United Kingdom \\ ${ }^{4}$ Department of Histopathology, Kings College Hospital, London, United Kingdom
}

\begin{abstract}
This is a first case of mycotic aneurysm due to Streptococcus constellatus in a 58 years old male patient with diffuse large B cell lymphoma. A mycotic aneurysm is a rare but life threatening and potentially fatal sequela of bacteraemia. A 58-year male patient initially presented with sepsis after 7 days of chemotherapy for non hodgkin's lymphoma. No clear focus of sepsis was found. Blood culture grew Streptococcus constellatus. He received appropriate antibiotic and discharged well. He represented after 5 days with excruciating intrascapular and chest pain. This patient with haematology malignancy had radiologically, surgically and histologically confirmed mycotic aneurysm that involved ascending aorta which contained pseudoaneurysm. Patient was treated excision of the infected tissue and aortic graft, and long term antibiotics. This rare case highlights that the high clinical suspicion in patients presenting with possibly mycotic aneurysm is a critical tool in addition to perfect surgical treatment and medical treatment with adequate antibiotics. J Microbiol Infect Dis 2017; 7(4):213-216
\end{abstract}

Keywords: Mycotic aneurysm, blood stream infections, cancer, diffuse large B cell lymphoma

\section{INTRODUCTION}

Infection of the aortic aneurysm is a rare but serious complication (1-3\%) of pre-existing aortic aneurysm, which is usually caused by the bacteria [1,2]. Infected aneurysm, also called as mycotic aneurysm, first described by Sir William Osler from Philadelphia USA in 1885 AD, arising as a complication of infective endocarditis in 30 years old male from Montreal. This patient presented with four aneurysms of the aortic arch [3].

However this term is still used regardless of the aetiology of infected aneurysm. Infected aneurysm carries a high risk of mortality. They pose great challenge and difficult clinical problem to the vascular surgeons and other clinicians who are involved in the management of patient. Several causative agents of mycotic aneurysm such as Salmonella, Pasteurella multocida, Staphylococcus aureus, Streptococcus species, Enterococcus species, Mycobacterium tuberculosis, Clostridium species, Escherichia coli, Haemophilus influenza, Listeria monocytogenes, and Burkholderia pseudomallei have previously been reported in the literature [4-7]. Medical treatment alone is not sufficient to treat infected aneurysm due to rapid progression of pseudoaneurysm; surgical treatment is warranted to avoid aortic fistula, aneurysm rupture and bleeding [8, 9]. We report a first case of mycotic aneurysm without endocarditis due to Streptococcus constellatus in 58 years old male with diffuse large B cell lymphoma from East Sussex Healthcare Trust (ESHT), United Kingdom.

\section{CASE}

A 58 years old man presented to the ear nose and throat (ENT) department with hoarseness of voice for two months. His Computed Tomography (CT) scan (Figure 1) showed upper mediastinal lymphadenopathy. The patient had endobronchial ultrasound guided biopsy (EBUS), as it was difficult to access percutaneously, which confirmed diffuse large $B$ cell lymphoma (DLBCL). His staging positron emission tomography (PET/CT) showed disease

Correspondence: Dr Sunil Gupta, Department of Clinical Haematology, East Sussex Healthcare Trust, Hastings, TN37 7RD, United Kingdom

Email: sunilgupta2@nhs.net

Received: 17 May 2017 Accepted: 29 September 2017

Copyright (C JMID / Journal of Microbiology and Infectious Diseases 2017, All rights reserved 
in the chest only. He had no bone marrow involvement so it was stage I disease. He was started on standard chemotherapy regime Rituximab, Cyclophosphamide, Doxorubicin, Vincristine and Prednisolone (R-CHOP).

After 7 days of chemotherapy, he presented to the emergency department of ESHT with fever, rigors, and lethargy for the last four days. On examination, patient was alert and had temperature of $38.2{ }^{\circ} \mathrm{C}$, a heart rate of 87 beats/minute, respiratory rate of $16 /$ minute, a blood oxygenation saturation of $97 \%$ on 15 liter oxygen therapy and blood pressure of $128 / 70$ $\mathrm{mmHg}$. White cell count $\left(14.4 \times 10^{9} / \mathrm{L}\right)$, neutrophil count $\left(12.4 \times 10^{9} / \mathrm{L}\right)$ and C-reactive protein $(119 \mathrm{mg} / \mathrm{L})$ were elevated. He had no clear focus of infection. His initial two sets of blood cultures remained sterile, and echocardiogram showed no evidence of vegetation. He was treated empirically with piperacillin/tazobactam for 48 hours, but when the fever persisted, piperacillin/tazobactam was switched over to Meropenem and Vancomycin. His third blood culture which was taken after 5 days of admission grew Streptococcus constellatus which was sensitive to amoxicillin, penicillin, clindamycin, linezolid, teicoplanin and vancomycin. Gradually his inflammatory markers improved and he felt much better. He received intravenous antibiotic for 8 days in total. He was discharged on oral amoxicillin/clavulanic acid for 5 more days. On the last day of his oral antibiotic treatment, he represented to the emergency department with excruciating chest and interscapular region pain for the last three days which was not resolved with analgesia. On admission, his temperature was $35.5{ }^{\circ} \mathrm{C}$, while heart rate, respiratory rate and blood pressure were within normal range. He had urgent CT scan of the chest which showed false aneurysm in the ascending aorta in the region adjacent to the necrotic mediastinal lymph node (Figure 2, 3). CT findings were suggestive of mycotic aneurysm. The patient was transferred urgently to the cardiothoracic department at the tertiary center. He underwent emergency surgery and had aortic wall rupture repair. Histology result from ruptured aortic wall showed heavy infiltration with neutrophils (Figure 4), and also confirmed Gram positive cocci, presumably Streptococcus constellatus (Figure 5). Unfortunately the tissue was not sent for culture.
The patient had full recovery post-operatively and received six weeks of intravenous vancomycin. He then completed rest of the 6 cycles of R-CHOP. He was in PET negative remission 6 months later and remained in good health till the report.

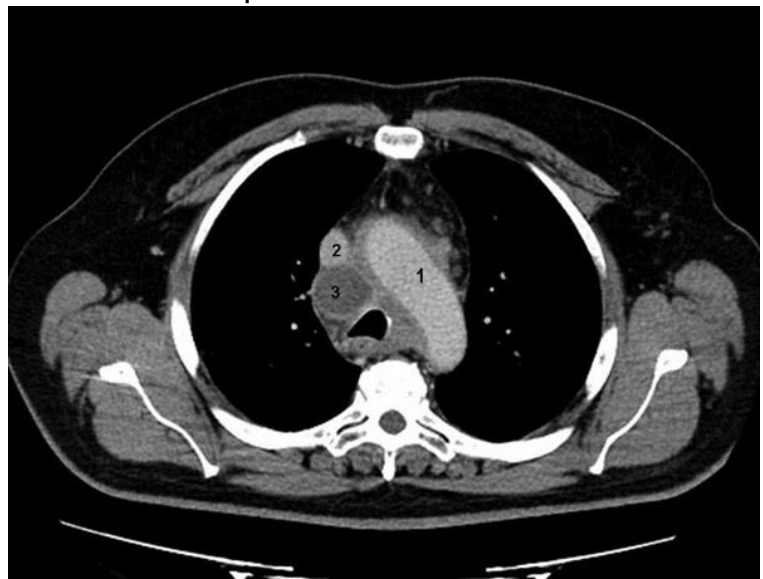

Figure1. CT shows upper mediastinal lymphadenopathy.

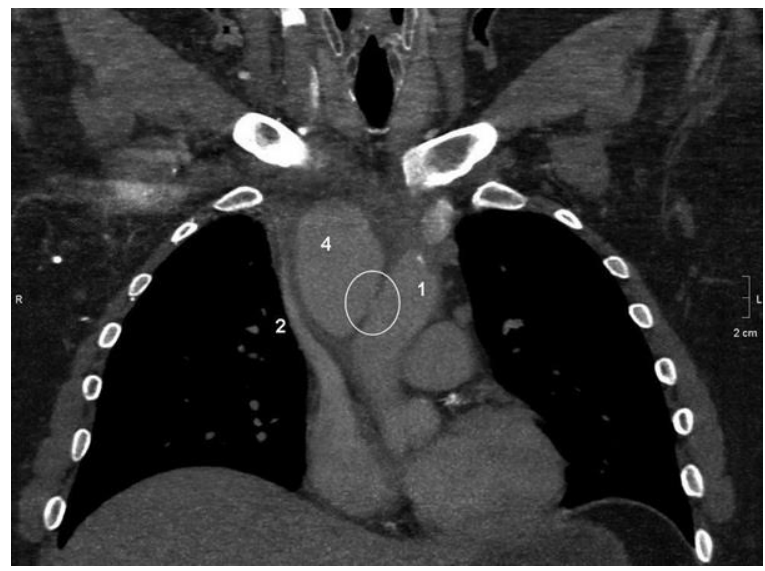

Figure2. CT scan of the chest which showed false aneurysm in the ascending aorta in the region adjacent to the necrotic mediastinal lymph node.

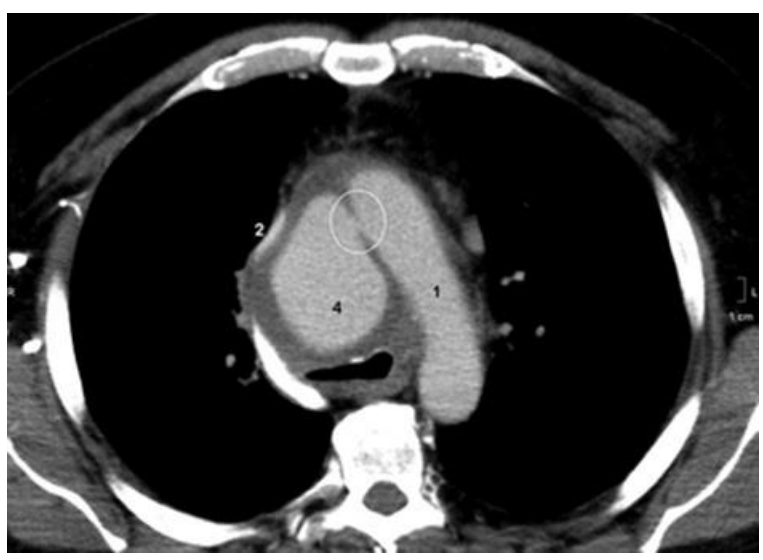


Figure3. CT scan of the chest which showed false aneurysm in the ascending aorta in the region adjacent to the necrotic mediastinal lymph node.

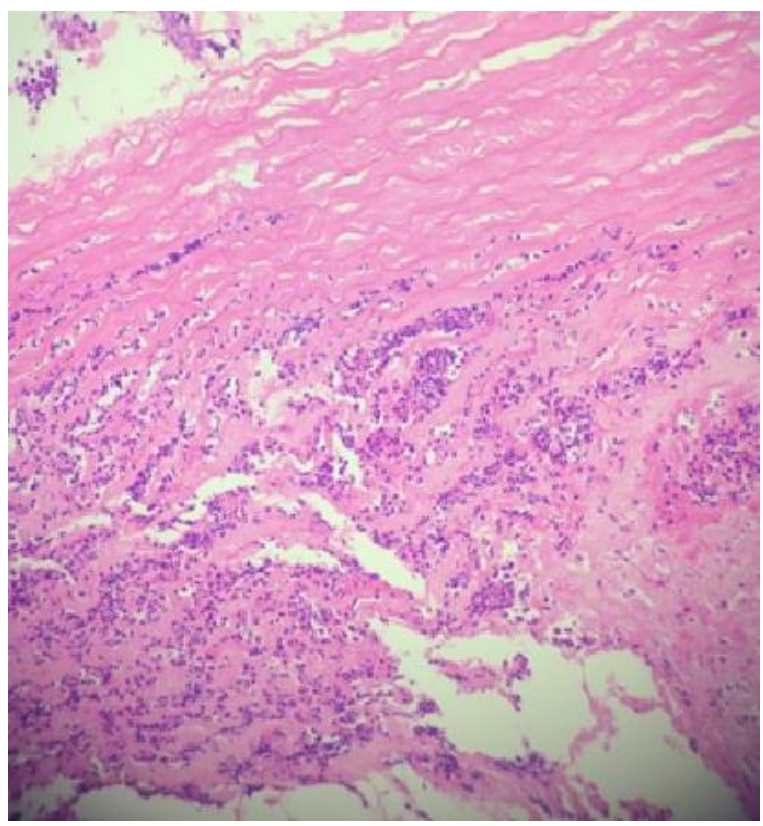

Figure 4. Histology result from ruptured aortic wall showed heavy infiltration with neutrophils.

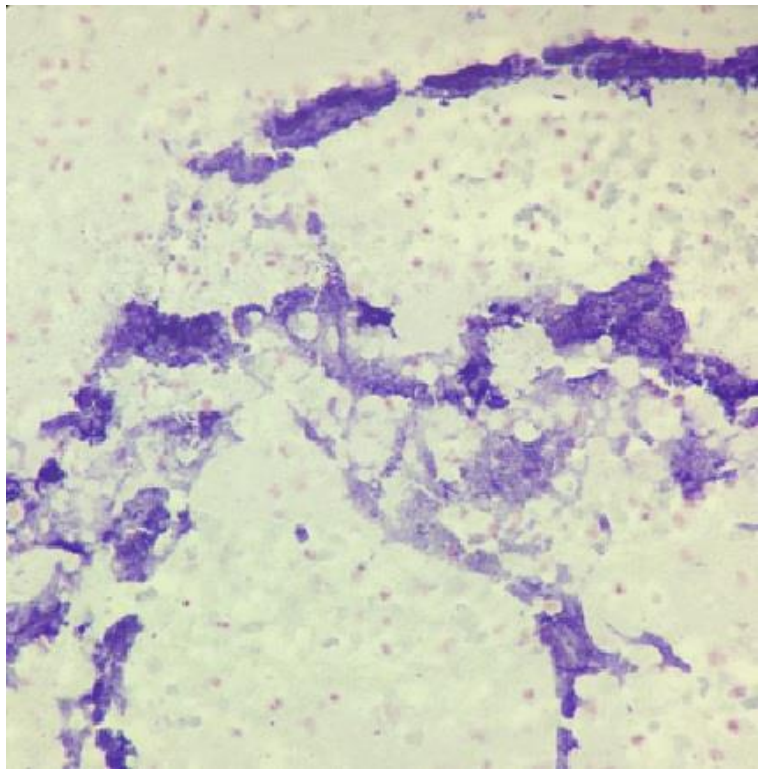

Figure 5. Histology result shows Grams positive cocci.

\section{DISCUSSION}

Mycotic or infected aneurysm is usually a bacterial infection in the wall of an artery with formation of a blind, saccular out-pouching which engulfs the entire arterial lumen. Delay in the diagnosis or delay in the treatment or no treatment often result in severe sepsis, spontaneous arterial rupture and fatal outcome [10]. Most cases of mycotic aneurysm are of haematogenous spread of septic emboli usually from infective endocarditis, or infection of already existing aneurysm, local spread from adjacent infectious source such as gastrointestinal tract infection, and direct introduction of infectious agent following a penetrating trauma or intravascular cannulation or intravenous drug abuse or cellulitis, or wound infection or dental extraction. Other possible cause of infected aneurysm, most common in aorta, is blood stream infection leading to infection of atheromatous plague and ultimately resulting in to the destruction of the wall and aneurysm formation $[4,9,10]$. In this interesting first case of mycotic aneurysm due to Streptococcus constellatus in haematology patient, we postulate that patient had contracted the bacteria during his professional work as a dentist. EBUS as part of his diagnostic pathway played a significant role in invading the infection in the right para-tracheal lymph nodes, which in turn possibly involved the aortic arch. The lymph node became necrotic on chemotherapy which helped creating a narrow connection between the lymph node and the aortic arch where a micro-abscess formed. This whole picture looked like an aortic pseudo-aneurysm. The early clinical manifestations of infected aortic aneurysm such as signs of system sepsis without any clear source may not cause suspicion about this disease. In this case, patient received treatment for sepsis of unknown origin during first admission. However, the clinicians suspected infected aneurysm during readmission when patient presented with intrascapular and chest pain and CT confirmed the diagnosis of the mycotic aneurysm. Histology report on Aortic tissue, which was taken during the surgical intervention, showed heavy infiltration with neutrophils and Gram positive cocci. Gram positive cocci are possibly Streptococcus constellatus and seeded to the pre-existing aortic aneurysm during bacteraemic episode. Streptococcus constellatus is a Gram positive cocci, non-spore forming organism. It is a species of Streptococcus milleri group and it is a part of the normal flora in the oral cavity, respiratory tract, urogenital region and gastrointestinal tract. Clinically this bacterium is 
associated with micro abscess formation in the upper body and respiratory tract and causes bacteraemia [11]. Streptococcus constellatus has been reported the cause of mycotic aneurysm in one case report in the English literature but not in the haematology patient [12].

Gold standard management consists of empirical broad spectrum antibiotics and immediate surgical intervention such as debridement of infected tissues, and artificial graft $[4,5]$. Every effort should be made to identify the causative agent of mycotic aneurysm. Appropriate treatment should be started after availability of the microbiology culture results. Duration of antibiotic treatment remains unclear and debatable. However, several previous published reports suggested a minimum of 6 weeks of intravenous antibiotics and oral regimen for another six weeks, and In Salmonella infection at least six months of antibiotic therapy is advocated. Some studies also suggested life course of antibiotics in individual cases $[4,13,14]$. Our patient received six weeks of intravenous vancomycin as Streptococcus constellatus was sensitive to vancomycin.

In conclusion, infected aortic aneurysm due to Streptococcus constellatus is extremely rare. Streptococcus constellatus positive blood cultures should incite the physicians to look for hidden abscesses if the symptoms do not improve. The definitive treatment of mycotic aneurysm in haematology patients consists of high clinical suspicion, prompt diagnosis, and initiation of the broad spectrum antimicrobial therapy which covers all possible causative agents and surgical intervention for example complete debridement of the infected tissues.

\section{ACKNOWLEDGMENTS}

Conflict of interest: The authors declare no personal or financial conflict of interest.

Funding disclosure: Non to declare
2. Müller BT, Wegener OR, Grabitz K, Pillny M, Thomas L, Sandmann W. Mycotic aneurysms of the thoracic and abdominal aorta and iliac arteries: experience with anatomic and extra-anatomic repair in 33 cases. J Vasc Surg 2001; 33: 106-113.

3. Osler W. The Gulstonian Lectures, on Malignant Endocarditis.Br Med J 1885 Mar 7; 1(1262): 467-470.

4. Laohapensang K, Aworn S, Orrapi S, Rutherford RB. Management of the infected aortoiliac aneurysms. Ann Vasc Dis 2012; 5: 334-341.

5. Koelemay MJ. Pasteurella multocida infection, a rare cause of mycotic abdominal aortic aneurysm. J Vasc Surg 2009; 50: 1496-1498.

6. Suarez CJ, Glover WA, Cowan J, Smith A, Clarridge JE $3^{\text {rd }}$. Mycotic aneurysm of the abdominal aorta caused by Haemophilus influenzae type f. J Med Microbiol 2013; 62: 658-660.

7. Murphy K, Al-Jundi W, Nawaz S. Mycotic aneurysms of the abdominal aorta due to Listeria monocytogenes. Int J Surg Case Rep 2013; 4: 626628.

8. Patel S, Johnston KW. Classification and management of mycotic aneurysms. Surg Gynecol Obstet 1977; 144: 691-694.

9. Dolapoglu A, de la Cruz KI, Coselli JS. Management of a Mycotic Thoracoabdominal Aortic Aneurysm Involving the Celiac Artery. Tex Heart Inst J 2016; 43: 528-530.

10. Lee W, Mossop P, Little A, et al. Infected (Mycotic) Aneurysms: Spectrum of Imaging Appearances and Management. RadioGraphics 2008; 28: 1853-1868.

11. Akuzawa N, Hatori T, Kitahara Y, Kurabayashi M. Multiple liver abscesses and bacteremia caused by Streptococcus constellatus infection: a case report. Clin Case Rep 2016; 5: 69-74.

12. Chiang WC, Tsai JC, Chen SY, et al. Mycotic Aneurysm Caused by Streptococcus constellatus subsp. constellatus. J Clin Microbiol 2004; 42: 1826 1828.

13. Ting AC, Cheng SW, Ho P, Poon JT. Endovascular stent graft repair for infected thoracic aortic pseudoaneurysms-a durable option? J Vasc Surg 2006; 44: 701-705.

14. Lee KH, Won JY, Lee DY, et al. Stent-graft treatment of infected aortic and arterial aneurysms. J Endovasc Ther 2006; 13:338-345.

\section{REFERENCES}

1. Alonso M, Caeiro S, Cachaldora J, Segura R. Infected abdominal aortic aneurysm: in situ replacement with cryopreserved arterial homograft. J Cardiovasc Surg (Torino) 1997; 38: 371-375. 